\section{Social preference in the guinea pig as a function of social rearing conditions and age at separation from the mother ${ }^{1}$}

\author{
Z. MICHAEL NAGY and JAMES $R$. \\ MISANIN, Susquehanna University, \\ Selinsgrove, $\mathrm{Pa} .17870$
}

To determine the effects of social rearing conditions and age at separation from the mother upon later social preferences, 120 guinea pigs were divided at birth into two basic groups, communals and isolates. Within the communal conditions, five groups of Ss remained with other guinea pigs throughout the experiment, but the mother was removed when $S$ s were 0, 4, 8,16, or 30 days of age. Within the isolate condition, $S$ s were separated from both the mother and other guinea pigs at $0,4,8,16$, or 30 days of age and remained isolated throughout the experiment until testing. At 135 days of age, social preference was tested in a tilt-cage apparatus for 6 consecutive days, 15 min daily. One end of the tilt cage contained another guinea pig, while the other end was empty. Results showed that preference for another guinea pig was reliably greater for the communally reared Ss than for those beginning isolation at any age. The age at separation from the mother had little effect upon social-preference scores for either rearing condition.

Several recent studies have reported that young guinea pigs form attachments or preferences to inanimate moving objects (Shipley, 1963; Sluckin, 1968; Sluckin \& Fullerton, 1969) and later prefer these objects to either unfamiliar objects or nothing when offered a choice in an approach situation. It has been suggested that these attachments may be regarded as examples of mammalian imprinting (Shipley, 1963; Sluckin, 1968), similar to imprinting found with many precocial birds. Earlier reports (Gray, 1958; Hess, 1959) have also suggested that isolated guinea pigs would imprint on humans.

In several of these studies (Sluckin, 1968; Sluckin \& Fullerton, 1969), it has been assumed that these attachments take place even in animals that have already formed strong attachments to the mother. However, none of these studies have presented the choice between the imprinting stimulus and the mother to animals given early exposure to an imprinting stimulus. Although not directly investigating imprinting, Harper (1968) has reported that guinea pigs isolated from birth showed greater approach to a strange guinea pig in a preference test than did mother- and communally-reared animals. This finding would not be expected if one assumed that imprinting had taken place in the nonisolated guinea pigs.

The purpose of the present study was twofold. The first purpose was to determine if the presence or absence of the mother had any effect upon later social preference for another guinea pig of animals maintained in communal conditions until testing. The second purpose was to determine if a critical period existed-during the early life of the guinea pig when social attachments were most easily or strongly formed. In this case, animals were separated from both the mother and other guinea pigs at ages ranging from $0-30$ days of age, followed by isolation until testing.

\section{SUBJECTS}

The Ss were 60 male and 60 female Hartley guinea pigs born and reared in the psychology department vivarium.

\section{APPARATUS}

The apparatus consisted of a straight alley, $30 \times 10 \times 12$ in., constructed of $1 / 2$-in. plywood painted flat black. At each end of the alley was a $7 \frac{112}{12} 10 \times 12$ in. compartment separated from the alley by transparent Plexiglas. The floor of the alley was constructed of $1 / 4$-in. wire mesh mounted on a central axle 15 in. from either end of the alley. A microswitch, mechanically depressed by the weight of the animal, operated a timing circuit that recorded the time spent on either side of the central axle. A shielded light source was placed $2 \mathrm{ft}$ above the center of the alley and provided a low level of illumination.

\section{PROCEDURE}

At birth, the guinea pigs were divided into 10 groups of six males and six females. Five groups of animals (communals) had the mother removed from the cage when the pups were 0 (a few hours after birth), $4,8,16$, or 30 days of age. These groups then remained with one to three animals of the same sex and age, although not always littermates, until testing. The other five groups (isolates) were separated from both the mother and other animals beginning at $0,4,8,16$, or 30 days of age and remained in isolation until testing. Social preference tests began at $135 \pm 10$ days of age. During the interval until testing, the animals were maintained on an ad lib diet of Purina Guinea Pig Lab Chow and water. Each S also received a daily supplement of $10-15 \mathrm{~g}$ of endive and was weighed daily. A 12-h light-dark cycle was used and the temperature was maintained at $72 \pm 2^{\circ} \mathrm{F}$. Both the isolate and communal groups were housed in Wahmann wire-cloth cages, $16 \times 10 \times 7$ in. Isolates could hear and smell other guinea pigs, but could not see them.

The preference test consisted of placing each animal individually into the center of the alley, facing a side wall. Upon placement in the apparatus, electric timers were started to record time spent in each half of the apparatus as well as the number of center crossings. Each trial lasted 15 min and one trial was given each day for 7 consecutive days.

On the first day of testing, the end compartments were empty in order to determine if Ss would exhibit a position preference for one end or the other, and also to allow Ss to adapt to the experimental test situation. On the second day of testing, a guinea pig of the same age and sex (communally reared) as $S$ being tested was placed in one of the end compartments, while the other compartment remained empty. The compartment into which the guinea pig was placed was at the end of the alley where each $S$ spent the least amount of time on the adaptation day. On each successive day, the position of the animal in the compartment was alternated in a counterbalanced fashion for each $S$.

RESULTS AND DISCUSSION

Figure 1 depicts the 6-day (excluding adaptation day) mean percentage of time spent on the side of the alley containing another guinea pig by isolate and communal $\mathrm{Ss}$ as a function of age at separation from the mother, or weaning

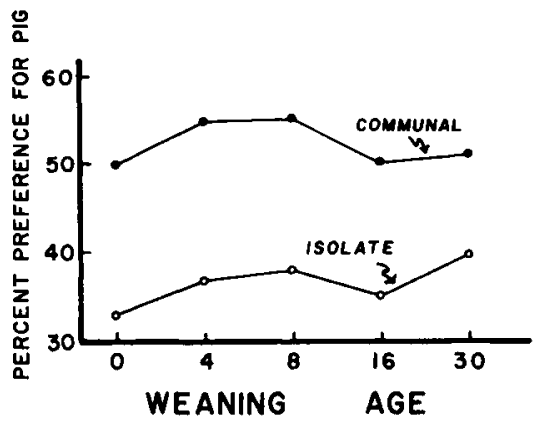

Fig. 1. Mean percentage of time spent on side of apparatus near another guinea pig for communal and isolate reared Ss as a function of weaning age. At these ages, communal Ss were separated from the mother, while the isolate Ss were separated from both the mother and all littermates. 
age. At these ages, isolate $S s$ began complete isolation (visual and tactual), while communal Ss remained with other age-mates of the same sex. Animals reared with other guinea pigs spent about $50 \%$ of the time in the half of the alley containing another guinea pig, while those beginning isolation between 0-30 days of age spent only about $35 \%$ of their time on the side containing another guinea pig. An analysis of variance revealed this difference to be highly reliable $(F=38.81 ; \quad d f=1 / 100$; $\mathrm{p}<.0005$ ). However, the age at which the mother was removed from the communal groups and the age at which the isolate groups began isolation had no apparent effect upon preference time $(F=0.85$; $\mathrm{df}=4 / 100$ ). The interaction of Rearing Conditions by Weaning Age was also not reliable $(F=0.33 ; \mathrm{df}=4 / 100)$. Preference time for another guinea pig increased for all groups over test days $(\mathrm{F}=6.64$; $\mathrm{df}=2 / 200 ; \mathrm{p}<.005)$ and males showed a higher score than did females $(F=4.39$; $\mathrm{df}=1 / 100 ; \mathrm{p}<.05)$.

A separate analysis of variance showed that there were no differences in the number of crossings between any of the groups tested, i.e., all groups explored both sides of the apparatus about equally. Activity was low for all groups, with the mean number of crossings per day ranging from 10 to 14 for all groups.

Freezing behavior, or the number of animals that did not move from where they were placed at the start of a trial, was also analyzed, since this type of behavior may be an indicator of emotionality in the guinea pig. Guinea pigs that did not move on any of the 6 test days were dropped from the study and replaced with other, similarly reared animals that were active on at least one of the trials. Of those discarded for freezing on all days, two Ss were reared in isolation, while eight were reared communally. Of the eight communals, five were in the group separated from the mother at 30 days of age. When the groups were completed with animals that showed activity or preference scores on at least 1 test day, the data were then examined for freezing behavior as a function of rearing conditions and test days. Figure 2 shows the percentage of isolate and communal animals freezing on the adaptation day and the first day of testing. On the adaptation day, when both end compartments were empty, 1 of 60 Ss reared in isolation failed to explore the apparatus. In contrast, 10 of $60 \mathrm{Ss}$ communally reared froze during the trial $\left(\chi^{2}=6.40 ; \mathrm{df}=1 ; \mathrm{p}<.02\right)$. On the first test day, when a guinea pig was present in one end compartment, the number of isolate Ss freezing increased to 13, while the communals showing no activity decreased to 8 . However, this

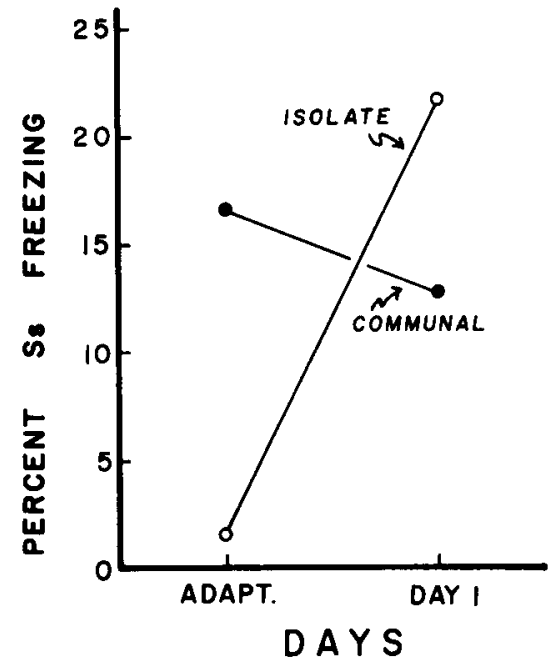

Fig. 2. Percentage of isolate and communal Ss freezing (failing to move during test session) on the adaptation day and first day of preference testing.

difference was not reliable, nor were any differences in freezing behavior between the isolate and communal Ss reliable over the remaining test days. The percentage of Ss freezing remained about the same over the remaining days as on the first day a guinea pig was present in the end compartment.

The results of the present study clearly show that social rearing conditions are a major factor in determining later approach to another guinea pig. While the tendency in group-reared Ss to approach and be near another guinea pig was not strong, it was greater there than in the isolate-reared $\mathrm{Ss}$, which tended to avoid being near another guinea pig. The results also show that for communally reared animals, the presence or absence of the mother made little difference in later preference scores. Most important, the present data suggest that there is no critical period in the early life of the guinea pig in which social preferences are most strongly formed. There was no reliable difference in later preferences between groups isolated at birth and those remaining with the mother and littermates through the first 30 days. From these data, it does not appear that guinea pigs imprint, or, if they do, imprinting is very weak and easily inhibited by social isolation.

Freezing behavior appears to be a useful index of emotionality for the guinea pig. This type of response has been reported to occur when the guinea pig is placed into an open-field situation (Tobach \& Gold, 1966). In the present study, isolated guinea pigs displayed almost no freezing behavior when placed in the apparatus with no animal present in the end compartment. However, when a strange guinea pig was placed in the end compartment the following day, the number of isolate animals freezing increased greatly. In contrast, a greater number of communal animals froze on the adaptation day than did isolates. This was the first time that the communals had been without the presence of other guinea pigs, and, apparently, this was reflected in the freezing behavior. On the first test day, when a strange guinea pig was in one of the end compartments, freezing behavior declined slightly and remained at that level over the remaining test days.

The present data contradict the findings reported by Harper (1968), who demonstrated that both male and female isolate guinea pigs approached and made contact with a tethered stimulus guinea pig more than did communally reared animals. In the present study, isolate animals clearly avoided a strange guinea pig more than did communally reared Ss. It is possible that the discrepancy in results was due to the test situation. In the present study, it was not possible for Ss to make physical contact with the object animal, whereas contact was possible in Harper's study. Secondly, in the present study, the animal placed in the end compartment tended to remain rather immobile throughout the test sessions, whereas this was probably not true with the tethered object animal. Thus, it is possible that movement is the stimulus causing approach behavior rather than the presence of the object animal per se. Indeed, in all of the studies claiming imprinting in guinea pigs (Shipley, 1963; Sluckin, 1968; Sluckin \& Fullerton, 1969), the imprinting objects were in motion both at the time of early exposure and during the test situations. However, this cannot be definitely concluded without further direct tests. The Harper study and the present one are in agreement, in general, in the finding that communally reared Ss had a greater tendency to freeze than did isolates, although this was true only on the adaptation day in the present study. When a guinea pig was present in the end compartment, there was no reliable difference in freezing behavior between isolates and communals.

In conclusion, the present results do not lend support to the hypothesis that guinea pigs imprint to objects or other guinea pigs as do precocial birds. Instead, they appear to develop preferences or attachments to objects to which they have been continually exposed, as has been reported for other mammals (Cairns, 1966).

REFERENCES

CAIRNS, R. B. Attachment behavior in 
mammals. Psychological Review, 1966, 73, 409-426.

GRAY, P. H. Theory and evidence of imprinting in human infants. Joumal of Psychology, $1958,46,155-166$

HARPER, L. V. The effects of isolation from birth on the social behaviour of guinea pigs in adulthood. Animal Behaviour, 1968, 16 58-64.

HESS, E. H. Imprinting. Science, 1959, 130, 133-141.

SHIPLEY, W. U. The demonstration in the domestic guinea pig of a process resembling classical imprinting. Animal Behaviour, 1963, $11,470-474$.
SLUCKIN, W. Imprinting in guinea pigs. Nature, $1968,220,1148$

SLUCKIN, W., \& FULLERTON, C. Attachments of infant guinea pigs. Psychonomic Science, $1969,17,179-180$.

TOBACH, E., \& GOLD, P. S. Behavior of the guinea pig in the open-field situation. Psychological Reports, 1966, 18, 415-425.

\section{NOTE}

1. This research was conducted by the authors at Princeton University in 1966 and was supported in part by NIMH Grant M-1562 to B. A. Campbell.

\section{Work rate with noncontingent supplement of the reward ${ }^{1}$}

PETER R. HARLEY, University of Oregon, Eugene, Oreg. 97403, and ALFRED E. ETAUGH, Bradley University, Peoria, Ill. 61606

Rats were trained to work for food on a VI 1-min schedule. During the operant sessions, a buzzer sounded throughout intervals in which free pellets were delivered every 30 sec. Neither the appearance nor continuation of this superimposed condition altered established rates. However, upon buzzer-free-food termination, rates were depressed and became less regular.

Intuitively, it seems that the rate of a simple operant response would be subject to change with variations in the animal's general emotional state. Estes \& Skinner (1941) made a convincing case for this notion in experiments where they presented a tone for several minutes during the stable emission of an operant and then terminated the signal with a foot shock to the rat. Ultimately, the rat stopped responding in the presence of the tone and resumed only after the shock. The flat spot in the cumulative record is now known as a conditioned emotional response, or the effect of conditioned suppression (Hunt \& Brady, 1951). The signal may be thought of as a conditioned stimulus (CS), and the unavoidable shock, as an unconditioned stimulus (US).

Looking for a complement to the conditioned-suppression paradigm, we substituted food for foot shock in the above procedure. Brady (1961) had seen rate facilitation during a signal for upcoming intracranial reward, but Azrin \& Hake (1969) have - reported establishing suppression during a CS followed by water, food, or rewarding intracranial stimulation. Since we were unable to observe either suppression or facilitation when we delivered a single food pellet after a 30 -sec audible buzz to an animal working for these pellets, we deviated somewhat from the usual approach in hope of strengthening the effect of the CS. In these explorations, a serendipitous observation has suggested itself as a matter of general psychological interest.

\section{METHOD}

Four rats were kept at $80 \%$ of their ad lib weights in Cohen constant-weight cages for 2 weeks prior to their first experimental sessions. During these 2 weeks, all animals were habituated to the Skinner box and magazine trained, while two of the rats ( 3 and 6 ) were given 15 Pavlovian sessions. Pavlovian treatments were separated by as little as $45 \mathrm{~min}$ and as much as 3 days. In these treatments, all four rats were in their closely apposed home cages and a buzzer was sounded for $5 \mathrm{~min}$. Rats 3 and 6 were automatically presented a $97-\mathrm{mg}$ pellet every $30 \mathrm{sec}$ while the other two rats ( 2 and 4 ) received no food. At the end of these 2 weeks, all rats were given daily hour-long periods in a Skinner box programmed to food-reward with a $45-\mathrm{mg}$ pellet on a variable-interval (VI) 1-min schedule. Two- to 8-min buzzer presentations, in which a free pellet was delivered every $30 \mathrm{sec}$, were superimposed on each operant session of Rat 6, which had had food in its home-cage buzz, and on those of Rat 2, which had had no food in its home-cage buzz. "Signaled free gratification" (SFG) appears to be the least problematic general label for the condition, since the actual contingencies for food presentation are never altered. Buzzer presentations in which no free pellets were presented, and where the VI 1-min schedule was also unaltered, were superimposed on the schedules of the other two rats ( 1 and 3 ). Rats 2 and 6 were given 17 daily sessions prior to extinction, and Rats 1 and 3 were given 9 such periods prior to extinction.

\section{RESULTS AND DISCUSSION}

No demonstrable rate facilitation was produced in any of these efforts, and neither was there any suppressive effect caused by the onset and continuation of the buzzer or the buzzer and free food. On the fourth day, however, Rat 2 began to show a mild post-SFG depression, which continued to appear in subsequent sessions. In the seventh running of Rat 2, an equipment failure resulted in the uninterrupted continuation of the buzzer-free-food condition for the last $35 \mathrm{~min}$ of the session. Figure 1 shows that introducing the SFG had no effect on response rate, in spite of the fact that pellet delivery during the buzzer was $300 \%$ of the nonbuzzer value. But in the eighth session, the termination of SFG in its usual brief amount resulted in a marked decrement in responding. Subsequent buzzer-free-food superimpositions had the same effect; i.e., onset and continuation of SFG did not alter the existing rate, but its termination caused the rat to slow down appreciably. In addition, responding after the post-SFG depression became less regular than usual. Reintroducing the buzzer-free-food during post-SFG depression restored the previous rate immediately.

In the ninth session for Rat 6, we allowed SFG to persist for approximately the last $35 \mathrm{~min}$, and again there was no rate change during this situation. In the 10th and 11 th sessions, Rat 6 failed to show the post-SFG depression produced by Rat 2 . Therefore, in its 12th session, SFG was again superimposed on the final $35 \mathrm{~min}$. There were no rate changes in the 13th period, but in the 14 th, the post-SFG depression appeared and was evident thereafter. However, both depression and subsequent rate irregularities for this animal were less pronounced than they were for Rat 2.

During extinction, as one might expect (Estes, 1943, 1948), the buzzer appeared to facilitate responding by Rats 2 and 6 , and did not affect Rats 3 and 4 .

We have not yet studied the acquisition of an operant in the continued presence of SFG, nor have we looked at a rat's 